\title{
La numérisation dans le domaine des soins ambulatoires
}

\author{
Tarja Zingg a ${ }^{\text {, Reinhold Sojer }}{ }^{\mathrm{b}}$, Fabian Röthlisberger ${ }^{\mathrm{c}}$ \\ ${ }^{a}$ Dr en sciences économiques, BA Communication Design, direction Lumina Health; ${ }^{b}$ Dr rer. biol. hum., responsable de la division Numérisation/eHealth, \\ $\mathrm{FMH} ;{ }^{c}$ Collaborateur scientifique à la division Numérisation/eHealth, $\mathrm{FMH}$
}

Le contexte suisse est favorable à la numérisation dans le domaine des soins ambulatoires, tant au niveau technique que sur le plan politique [1]. Pour la population, l'accès à Internet et l'utilisation de smartphones sont une évidence. Des infrastructures numériques comme le dossier électronique du patient (DEP) sont en cours de construction, et les prestataires et services numériques se multiplient dans le secteur de la santé. Les patientes et les patients tout comme le corps médical sont largement ouverts à l'évolution numérique dans les soins ambulatoires; or, 50\% seulement des cabinets médicaux en Suisse ont recours à des dossiers informatisés [2, 3].

Pourtant, la numérisation ne se fera pas toute seule dans le domaine ambulatoire. Il faut intervenir pour harmoniser les bases légales variant encore entre les cantons, développer des standards pour l'interopérabilité et créer des incitatifs financiers et non financiers. En outre, les patientes et les patients ainsi que les médecins doivent disposer des compétences appropriées afin de tirer le plus grand parti possible des applications numériques.

La FMH a dressé une vue d'ensemble des opportunités et des risques de la numérisation dans le domaine ambulatoire, conçue par des médecins à l'intention des médecins, et nous en reprenons ici les principaux éléments en détail ${ }^{1}$.

\section{Accès et utilisation d'Internet quasi généralisés}

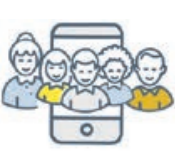
En 2017, 93\% des ménages avaient accès à Internet et près de $80 \%$ de la population suisse possédait un smartphone. Les conditions technologiques pour un accès généralisé et facilité aux services numériques de santé sont donc réunies [4].

Les services numériques de santé ont le vent en poupe

1 Cf. www.fmh.ch $\rightarrow$ Politique \& Thèmes $\rightarrow$ eHealth $\rightarrow$ Télémédecine $\rightarrow$ «Numérisation de la prise en charge médicale»

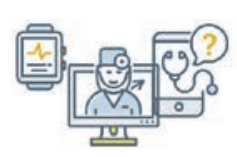

Les services numériques de santé sont de plus en plus demandés et utilisés. Il n'y a jamais eu autant de sites de santé et de cabinets médi- caux en ligne qu'aujourd'hui. Le carnet de vaccination est disponible en version numérique et la possibilité d'obtenir une prescription médicale par Internet ou par e-mail fait l'objet d'une demande et d'une offre croissantes. La majorité des patients souhaitent pouvoir communiquer avec leur médecin via messagerie instantanée ou par e-mail. Les applications mobiles et les objets connectés sont toujours plus ancrés dans le quotidien de la population; $44 \%$ de la population suisse utilise des applications pour l'entraînement et l'exercice physiques [3]. Le nombre des applications dans les catégories "Santé» et «Fitness et médecine» s'élève actuellement dans le monde à plus de 300000 [5]. L'offre continuera d'augmenter pour celles qui présenteront un intérêt manifeste pour les patients.

Prestataires et offres numériques en progression: 2,5 millions de contacts de patients par an

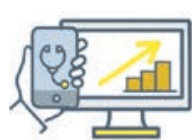

Diverses organisations de télémédecine proposent aujourd'hui des consultations sur les questions liées à la prévention, à la maladie, à la guérison, aux accidents, à la grossesse ou au soulagement de symptômes, et ce en tout lieu et en tout temps. Il suffit parfois d'une consultation approfondie par télémédecine pour prendre en charge correctement une légère affection. La consultation médicale en ligne bénéficie désormais d'une large approbation de la société. Aujourd'hui, 13\% des assurés ont choisi le modèle de télémédecine et les quatre principaux prestataires (Medgate, Medi24, Monvia, santé24) enregistrent ensemble quelque 2,5 millions de contacts de patients par an - une tendance à la hausse [6].

\section{Un accès élargi et facilité aux connaissances en matière de santé}

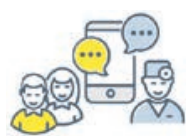

Les services numériques comme les sites de santé, les téléconsultations ou les applications de santé permettent d'accéder facilement, en tout lieu et en tout temps, à la promotion de la santé et à la prévention. Dans un pays où $93 \%$ des ménages disposent d'un 
accès Internet [4], cela représente un accès quasi généralisé aux connaissances actuelles en matière de santé. A l'avenir, pratiquement plus aucun patient ne consultera un médecin sans s'être préalablement informé de la qualité du praticien et de sa propre maladie sur Internet [7]. Toutefois, une partie de la population se sent dépassée par la numérisation: il convient donc de déployer des efforts particuliers pour accroître les compétences et la participation de ces personnes [8].

\section{Les possibilités existantes restent trop peu mises à profit}

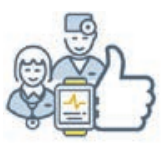

Malgré une attitude positive face aux services numériques de santé, la majorité des médecins y recourt encore peu. Ceux qui ont choisi de les intégrer dans leur quotidien professionnel cherchent notamment à améliorer les processus d'administration, de communication et d'information (surtout dans les grands cabinets), domaines où la valeur ajoutée est évidente. Près de la moitié des cabinets médicaux numérisent entièrement leurs dossiers médicaux [3]. En matière de soutien thérapeutique, les médecins utilisent les services numériques pour établir un meilleur diagnostic ou pour personnaliser le traitement (p. ex. ressources factuelles comme uptodate.com). Les applications numériques sont particulièrement attrayantes lorsque leur utilisation est aisée et lorsqu'elles contribuent à améliorer les processus de diagnostic et de traitement, à offrir des informations fiables ou à augmenter la flexibilité dans le temps et l'espace [2].

\section{La télémédecine a beaucoup de potentiel}

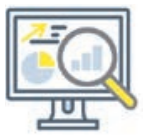

L'amélioration de l'efficacité et les économies de coûts obtenues grâce à la numérisation sont rapidement perceptibles dans les domaines de la gestion, de l'information et de la communication. Mais un potentiel d'économies et des améliorations de processus existent également dans les domaines diagnostique et thérapeutique, en particulier pour le traitement de troubles de la santé faciles à détecter et rapidement soignables, ou pour le traitement ou monitorage de certaines affections (p. ex. BPCO) et maladies chroniques, pour lesquelles une prise en charge au domicile du patient devient de plus en plus fréquente [9].

\section{Importance cruciale de l'interopérabilité des infrastructures}

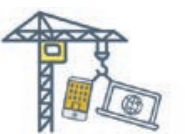

Dans un système de santé moderne, compatibilité et interopérabilité sont les maîtres-mots du fondement technique des infrastructures numériques.
La poursuite de leur développement coordonné aide à faire tomber les barrières et à augmenter les synergies et les gains d'efficacité. Seules la mise en réseau et l'évolution numérique du système de santé permettront d'accéder en temps réel aux nouvelles connaissances médicales et de les intégrer dans une prise en charge améliorée centrée sur le patient et le résultat [10].

\section{Atténuer les points faibles et minimiser les risques}

Même si la Stratégie Cybersanté Suisse 2.0 a jeté les nouvelles bases de la numérisation, plusieurs obstacles restent à surmonter dans le secteur ambulatoire [11] Il faut s'attaquer aux points faibles, notamment en matière de bases juridiques, de protection des données, d'interopérabilité et de prise en compte dans le tarif, et minimiser les risques liés à l'absence d'efficacité et au manque de compétences. Cela permettrait d'accélérer la numérisation parfois hésitante, de réduire les inefficacités et de maîtriser les coûts.

Voici une sélection des points faibles et des risques de la numérisation dans le domaine ambulatoire:

\section{La protection et la sécurité des données ne sont pas encore clairement définies}

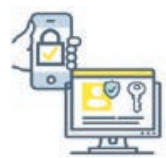

La protection et la sécurité des données sont des sujets délicats et émotionnels, car les données des patients sont très sensibles. C'est pourquoi les services et les applications de télémédecine sont soumis à une protection renforcée. Les données des patients ne devraient être accessibles qu'aux médecins, thérapeutes et employés impliqués et être protégées contre l'accès de tiers, qui ne doivent pas pouvoir les consulter. En effet, la transmission sécurisée des données n'est pas encore une évidence aujourd'hui. D'un autre côté, l'analyse d'importants volumes de données complexes comportant des informations pertinentes pour le domaine de la santé présente un énorme potentiel pour faire avancer la recherche et la médecine de manière plus rapide et interdisciplinaire [12, 13].

\section{Prise en compte insuffisante dans le tarif}

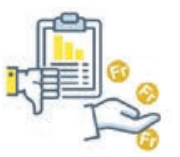

Les modèles d'indemnisation, la facturation et le remboursement de la télémédecine, mais aussi les positions TARMED pour les différentes prestations relevant du numérique, sont autant de questions qui ne sont pas encore réglées. En effet, les positions tarifaires applicables aujourd'hui couvrent ce domaine de manière incomplète et insuffisante. Le tarif 
TARMED n'incite pas à recourir à la télémédecine, car aucune position ne permet de la facturer. Or, pour les petits cabinets médicaux, l'absence de systèmes d'indemnisation ou de prise en compte tarifaire adéquate constitue un obstacle à l'investissement et à l'intégration de services numériques de santé [2].

\section{Des bases légales hétérogènes qui varient d'un canton à l'autre}

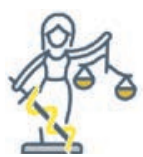

Les bases légales qui encadrent la numérisation dans le domaine ambulatoire sont très différentes d'un canton à l'autre et souvent en retard par rapport à la situation sur le terrain. Un premier examen a montré par exemple que les prestations de télémédecine étaient tout à fait possibles en Suisse et a confirmé ces différences cantonales. De nombreux cantons (p. ex. BL, ZH) interdisent la prise en charge exclusive de patients via la télémédecine ou la soumettent expressément à une autorisation [14].

\section{La situation sur le terrain est plus clairvoyante que le cadre légal}

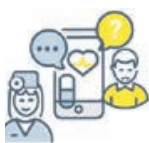

Sur le terrain, ce sont surtout les jeunes médecins qui utilisent et proposent des services numériques de santé pour répondre à la demande [2]; mais ils le font dans des conditions qui sont aujourd'hui loin d'être optimales, notamment concernant l'indemnisation, le cadre légal et la protection des données. La majorité des patients explore de plus en plus les possibilités du numérique dans leur vie quotidienne et réclame des services numériques également pour leur santé. Dans ce domaine, leurs besoins englobent désormais aussi une communication et des téléconsultations via messagerie instantanée ou par e-mail, un système électronique pour les transferts de patients, des applications de santé et des objets connectés [3].

\section{Besoin d'analyser l'efficacité et de fixer les principes d'évaluation}

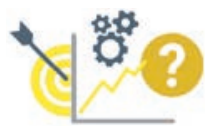

Les médecins installés sont prêts à la numérisation pour leur cabinet si le rapport coût/efficacité présente un bilan positif et que les avantages pour les patients sont avérés [2]. La preuve de l'efficacité et de l'utilité des services numériques de santé, notamment dans le domaine thérapeutique, est encore insuffisante [15]. Mais la pression croissante sur les coûts va accentuer le besoin d'orienter ces services à l'évidence médicale. Pour cela, une condition essentielle à leur généralisation consistera à évaluer méthodiquement les possibilités de prise en charge de la télémédecine [16]. Afin d'encourager leur acceptation, il est indispensable que les avantages administratifs, mais aussi et surtout diagnostiques et thérapeutiques, soient rapidement perceptibles, car, en définitive, tout gain d'efficacité permet d'accorder davantage de temps aux patients.

\section{Apprivoiser la numérisation, au même rythme}

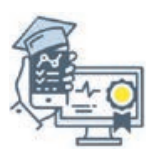

La numérisation exige acceptation et objectifs communs. Les différences d'affinité et de compétence se traduisent aussi bien chez les médecins que chez les patients par un accès différent et une utilisation différente des services numériques. Les opportunités de cette transformation seront réduites si les compétences numériques du corps médical et des patients ne sont pas encouragées simultanément. Dans ce contexte, la formation pré- et postgraduée d'un côté et le change management, de l'autre, font partie des points stratégiques les plus importants $[8,11,17,18]$.

\section{Objectifs 2022 pour la numérisation dans le domaine ambulatoire}

La numérisation dans le domaine de la santé est une tendance de fond, souvent associée aux phénomènes de «blockchain», "cyber security» ou «big data». Mais l'évolution numérique dans le domaine ambulatoire ne doit pas nécessairement s'envisager dans de telles dimensions. Les possibilités et les répercussions de la numérisation ont mené, et mènent encore, à des changements positifs majeurs dans le secteur ambulatoire, même par petites étapes pragmatiques (p. ex. dans les domaines de la gestion du diabète ou des vaccinations) [19, 20]. Ces initiatives devront toujours s'orienter vers l'amélioration des soins médicaux, les gains potentiels d'efficacité et l'augmentation de la sécurité des patients. Toutefois, les efforts déployés à petite échelle ne seront efficaces que si les conditions-cadres techniques et politiques et les incitatifs sont favorables.

Les objectifs 2022 pour la numérisation dans le domaine ambulatoire conjuguent facteurs de réussite et situation souhaitée à l'avenir. Pour leur élaboration, il a été inéluctable de réunir des points de vue différents dépendant chacun d'une perspective subjective. C'est pourquoi les appréciations de différents partenaires de santé ont été recueillies de sorte que les dix principaux messages des objectifs 2022 reflètent les opinions de tous les acteurs.

Il reste à définir les responsabilités pour la formulation prochaine et coordonnée des mesures en vue de la 
mise en œuvre des objectifs. Mais une chose est sûre: la réussite de la numérisation dans le domaine des soins ambulatoires dépend de la participation perti- nente des acteurs concernés, du corps médical et des patients à toutes les phases de cette entreprise.

\section{Numérisation de la prise en charge médicale}

Situation actuelle et objectifs 2022 conçus par des médecins à l'intention de médecins

\section{¡FMH}

\section{Objectifs 2022}

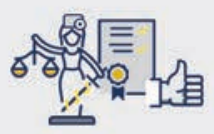

(1) Le cadre légal concernant l'offre, l'utilisation et l'indemnisation des services numériques de santé existe. II est harmonisé entre les cantons.

2 Les directives et les critères qualité de la télémédecine existent pour toute la Suisse et servent d'aide et d'orientation aux médecins et aux patients.

\section{Technologie}

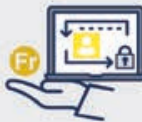

(3) La protection et la sécurité des données sont suffisamment garanties.

(4) Les incitatifs pour développer, produire et distribuer des logiciels de télémédecine à forte valeur ajoutée existent.

\section{Finances}

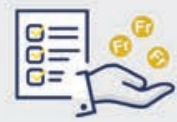

(5) Des modèles de rémunération appropriés garantissent l'indemnisation des prestations numériques de santé et la rémunération financière est réglementée.

\section{Offre et processus}

6 Les services numériques de santé permettent d'optimiser la prise en charge médicale et offrent une valeur ajoutée aux patients et aux médecins.

(7) Les services numériques viennent compléter les prestations médicales au sens d'une prise en charge plus efficace et plus sûre ; les interactions humaine et numérique sont en équilibre.

8 Les services numériques facilitent et améliorent les processus de gestion et d'administration, le déroulement de la relation médecin-patient et la communication entre les différents acteurs de la santé.

\section{Connaissances et appréciations}

Correspondance:
9 Les évolutions sociétales sont prises en compte dans l'intégration des services numériques ; les patients sont en mesure d'utiliser correctement ces services.

(10 Les médecins sont ouverts aux services numériques de santé, ils exploitent les avantages de la transformation numérique et sont en mesure d'évaluer, d'utiliser et de recommander des services appropriés. 